\title{
Changing trends in cytomegalovirus retinitis with highly active anti-retroviral therapy (HAART)
}

Over the past decade, AIDS-related CMV retinitis (CMVR) has been transformed from a blinding pre-terminal event to a manageable chronic condition. Highly active anti-retroviral therapy (HAART) refers to a combination of drugs incorporating both reverse transcriptase and protease inhibitors. This therapeutic approach can lead to a dramatic fall in HIV load, an increase in CD4 ${ }^{+}$T-lymphocyte count and partial immune reconstitution. This has led to a reduction in morbidity and mortality associated with AIDS. ${ }^{1}$

One consequence of HAART has been a remarkable fall in the numbers of new patients with CMVR. However, alteration in the natural history, presenting signs and coexisting uveitis have been described in a recent paper in Eye by Lee $e t a l .^{2}$ Their findings reflect the experience of ophthalmologists and physicians specialising in AIDS working in units who have access to HAART.

Partial immune reconstitution in the individual with established CMVR offers the hope of discontinuing maintenance anti-CMVR therapy. ${ }^{3}$ This is considered if an elevated $\mathrm{CD} 4^{+}$ cell count and low or undetectable HIV load is sustained. ${ }^{3}$ Komanduri et al. reported restoration of CMV-specific CD4 ${ }^{+}$lymphocyte response in individuals with CMVR following ganciclovir and HAART. ${ }^{4}$ Discontinuing antiCMV therapy reduces both drug toxicity and drug-resistant organisms. However, CMVR has been reported to occur despite HAART-induced elevation of $\mathrm{CD}^{+}{ }^{+}$lymphocyte count; therefore close monitoring is mandatory when anti-CMV therapy is stopped. ${ }^{5}$ Replacement of ganciclovir sustained-release implants in HAARTresponders may now be deferred until breakthrough infection has been confirmed.

Those individuals with established CMVR who fail to respond to HAART or who have suffered irreversible loss of specific cellmediated anti-CMV immunity despite increasing $\mathrm{CD}^{+}{ }^{+} \mathrm{T}$-lymphocyte counts present a major management challenge. Under these circumstances, extended life survival with both HAART-associated uveitis and CMVR breakthrough events are likely to have a negative impact on visual function over the longer term.

A combination of factors leads to HAART failure in approximately $20 \%$ of individuals due to poor compliance with the multi-drug regimen, drug toxicity, drug interactions and HIV drug resistance. Therefore, ophthalmologists and physicians with an interest in HIV medicine should look towards modifying and not stopping surveillance for CMVR in AIDS. Departures from the 'classic' presentation of CMVR have been described, including HAART-associated vitritis, and vitreo-macular traction syndrome. ${ }^{6,7}$ New antiCMV drugs, for example cidofovir, are also triggers of intraocular inflammation and the sequelae of posterior synechiae, cataract and vitreous haze are likely to impede fundus examination. This may lead to confusion in differentiating CMVR from other diagnoses including Toxoplasma retinochoroiditis and lymphoma. Under these circumstances intraocular fluid sampling for polymerase chain reaction studies shall be needed rather than relying on ophthalmoscopic diagnosis. Media haze also causes difficulty in recognising relapsing CMVR infection. Coexisting cystoid macular oedema may be a feature of active CMV retinitis and has also been observed with HAART-mediated intraocular inflammatory disease - the dilemma of treatment with periocular or systemic steroids for fear of exacerbating infection remains to be resolved.

It is possible that CMV viral load testing and monitoring cytokine levels might predict those individuals at greatest risk of developing CMVR or breakthrough infection following established disease. ${ }^{8}$ It is not clear how virological testing and the cost implications of this approach will influence the management of an ever-increasing pool of HIV-infected individuals in the future.

The dramatic impact of HAART in altering the natural history of CMVR has fuelled a growing sense of optimism amongst individuals with HIV infection and ophthalmologists alike,

\author{
B. Dhillon \\ K. Ramaesh \\ Princess Alexandra Eye \\ Pavilion \\ Edinburgh, UK \\ C. Leen \\ Regional Infectious Unit \\ Western General Hospital \\ Edinburgh, UK \\ B. Dhillon \\ Princess Alexandra Eye \\ Pavilion \\ Chalmers Street \\ Edinburgh EH3 9HA, UK
}


though it should be remembered that HAART does not cure HIV infection, merely controls progression of the disease. Premature claims that the CMVR 'epidemic' is over runs the risk of increasing the visual morbidity in AIDS, through delayed diagnosis, failure to recognise breakthrough infection and inadequate treatment of progressive CMVR. One consequence of prolonging both survival and quality of life by HAART in HIV infection is complacency in adhering to safe sex practices and harm reduction behaviour in 'at-risk' groups. Currently, Africa and Southeast Asia are experiencing the most rapid rise in HIV infection and AIDS, and for these individuals HAART is not affordable.

\section{References}

1. Palella FJ, Delaney KM, Moorman AC, Loveless MO, Fuhrer J, Satten GA, et al. Declining morbidity and mortality among patients with advanced human immunodeficiency virus infection. N Engl J Med 1998;338:853-60.

2. Lee V, Subak-Sharpe I, Shah S, Aitken C, Limb S, Pinching A. Changing trends in CMV retinitis with triple therapy. Eye 1999;13:59-64.
3. Vrabec TR, Baldassano VF, Whitecup SM. Discontinuation of maintenance therapy in patients with quiescent cytomegalovirus retinitis and elevated $\mathrm{CD} 4{ }^{+}$counts. Ophthalmology 1998;105:1259-64.

4. Komanduri KV, Viswanathan MN, Wieder ED, Schimudt DK, Bredt BM, Jacobson MA, McCune JM. Restoration of cytomegalovirus-specific $\mathrm{CD} 4{ }^{+} \mathrm{T}$-lymphocyte response after ganciclovir and highly active antiretroviral therapy in individuals infected with HIV-1. Nature Med 1998;4:953-6.

5. Pavan PR, O'Donnell JJ, Sattler F, Rao N, Owens S, Pollard R. Cytomegalovirus retinitis after initiation of highly active antiretroviral therapy. Lancet 1997;349:1443-5.

6. Canzano JC, Reed JB, Morse LS. Vitreomacular traction syndrome following highly active antiretroviral therapy in AIDS patient with cytomegalovirus retinitis. Retina 1998;18:443-7.

7. Herbort CP, Chave JP. Cicatrization of cytomegalovirus retinitis following introduction of highly active anti-retroviral therapy: uveitis as a possible indicator of good ocular prognosis. Graefes Arch Clin Exp Ophthalmol 1998;236:795-7.

8. Michelson S, Alcami J, Kim S-J. Human cytomegalovirus infection induces transcription and secretion of transforming growth factor beta 1. J Virol 1994;68:5730-7. 